\title{
Fitness, Strength and Body Composition during Weight Loss in Women with Clinically Severe Obesity: A Randomised Clinical Trial
}

\author{
Clint T. Miller ${ }^{a} \quad$ Steve F. Fraser ${ }^{a}$ d $\quad$ Steve E. Selig ${ }^{a} \quad$ Toni Rice $^{b, c}$ \\ Mariee Grimab, c Daniel J. van den Hoek ${ }^{d, e}$ Carolina Ika Sari ${ }^{c}$ \\ Gavin W. Lambert ${ }^{c, f}$ John B. Dixon ${ }^{b, f}$ \\ aSchool of Exercise and Nutrition Sciences, Deakin University, Geelong, VIC, Australia; \\ ${ }^{b}$ Clinical Obesity Research Laboratory, Baker Heart and Diabetes Institute, Melbourne, VIC, \\ Australia; ${ }^{\mathrm{C}} \mathrm{Human}$ Neurotransmitters and Clinical Obesity Research Laboratory, \\ Baker Heart and Diabetes Institute, Melbourne, VIC, Australia; ${ }^{d}$ Institute for \\ Physical Activity and Nutrition, Deakin University, Geelong, VIC, Australia; ${ }^{e}$ School of \\ Behavioural and Health Sciences, Australian Catholic University, Brisbane, QLD, Australia; \\ fIverson Health Innovation Research Institute and School of Health Sciences, \\ Swinburne University of Technology, Melbourne, VIC, Australia
}

Keywords

Obesity $\cdot$ Exercise training $\cdot$ Women $\cdot$ Fitness $\cdot$ Body composition

\begin{abstract}
Introduction: To determine whether combined exercise training with an energy-restricted diet leads to improved physical fitness and body composition when compared to energy restriction alone in free-living premenopausal women with clinically severe obesity. Methods: Sixty premenopausal women (BMI of $40.4 \pm 6.7$ ) were randomised to energy restriction only (ER) or to exercise plus energy restriction (EXER) for 12 months. Body composition and fitness were measured at baseline, 3, 6 and 12 months. Results: $\mathrm{VO}_{2}$ peak improved more for EXER compared to ER at 3 (mean difference \pm SEM $2.5 \pm 0.9 \mathrm{~mL} \cdot \mathrm{kg}^{-1} \cdot \mathrm{min}^{-1}, p=0.006$ ) and 6 (3.1 $\left.\pm 1.2 \mathrm{~mL} \cdot \mathrm{kg}^{-1} \cdot \mathrm{min}^{-1}, p=0.007\right)$ but not 12 months $\left(2.3 \pm 1.6 \mathrm{~mL} \cdot \mathrm{kg}^{-1} \cdot \mathrm{min}^{-1}, p=0.15\right)$. Muscle strength improved more for EXER compared to ER at all time points. No differences between groups for lean mass were observed at 12 months. Conclusion: Combining exercise training with an energy-restricted diet did not lead to greater aerobic power, total body mass, fat mass or limit lean body mass loss at 12 months when compared to energy restriction alone for premenopausal women with clinically severe obesity in free-living situations. Future research should aim to determine an effective lifestyle approach which can be applied in the community setting for this high-risk group.


Miller et al.: Exercise Training in Severe Obesity

\section{Introduction}

Women are more prone to clinically severe obesity (body mass index, BMI, $\geq 40$, or a BMI $\geq 35$ with obesity-related comorbidities) than men [1]. Individuals with classes II (BMI 35-39.9) and III obesity (BMI $\geq 40$ ) are at higher risk of developing metabolic and cardiovascular diseases when compared with class I obesity (BMI $\geq 30-34.9$ ) [2], and this places twice the burden on health care systems when compared with healthy weight adults [3]. Health care utilisation for cardiometabolic disorders does not fully account for the true cost of obesity. Increasing obesity and central adiposity lead to greater risks of developing obesityrelated disabilities and musculoskeletal injuries [2], which may negatively impact on occupational capacity, social engagements and activities of daily living. Individuals with severe obesity experience a feed-forward cycle of low exercise capacity, physical disability and breathlessness leading to physical inactivity, in turn leading to further weight gain [4] and progressive loss of physical function [5], resulting in frailty in later life [6]. Therefore, strategies to enhance the fitness of adults with clinically severe obesity are important for the prevention of later life physical disability.

Clinical practice guidelines suggest diet and exercise as first-line treatment strategies for overweight and obesity [7]. These interventions produce moderate weight loss in the short term, but the weight is often regained in the long term [8]. Much of the literature concerning energy restriction and physical activity interventions focus on adults who are categorised as having overweight or class I obesity [9]. Whilst exercise can limit the loss of lean body mass and improve physical function when added to energy-restricted diets in adults with class I obesity, exercise plus diet does not produce substantial additional weight loss when compared to diet alone $[8,10]$. Whether these findings apply to more severe classes of obesity has not yet been established and so was a focus of this study. There is some evidence that adaptations to exercise could be blunted in obesity [11] due to impaired skeletal muscle protein metabolism, muscle fibre typology [12], sympathetic nervous reactivity [13], brown adipose activation [14] and altered skeletal muscle mitochondrial content and function [15].

An appropriately designed exercise training programme can improve physical fitness and body composition $[10,16]$; however, despite the increasing prevalence and the unique challenges that adults with clinically severe obesity face, this population group has not received adequate attention in long-term clinical trials that focus on exercise training. Therefore, the main aim of this study was to investigate whether combined exercise training and an energy-restricted diet improved physical fitness and body composition, compared with an energy-restricted diet alone for women with clinically severe obesity under freeliving conditions.

\section{Materials and Methods}

Sixty women with clinically severe obesity were recruited for a 12-month, parallel group, randomised clinical trial from Melbourne, Australia, between September 2011 and June 2015 (Fig. 1). All data were available for analysis from November 2016. All participants provided written informed consent and obtained medical clearance prior to enrolling. The full study protocol has been published previously [17]; however, in short, the inclusion criteria were premenopausal women aged 18-50 years, with a BMI $\geq 40$ or a BMI $\geq 30$ with at least 1 obesity-related comorbidity. The exclusion criteria were unstable cardiovascular conditions, type 1 diabetes, active musculoskeletal conditions preventing exercise participation, pregnancy or planning pregnancy, weight loss greater than $5 \mathrm{~kg}$ in the past 3 months, using weight loss medications, previous bariatric surgery, medications which significantly influence weight 


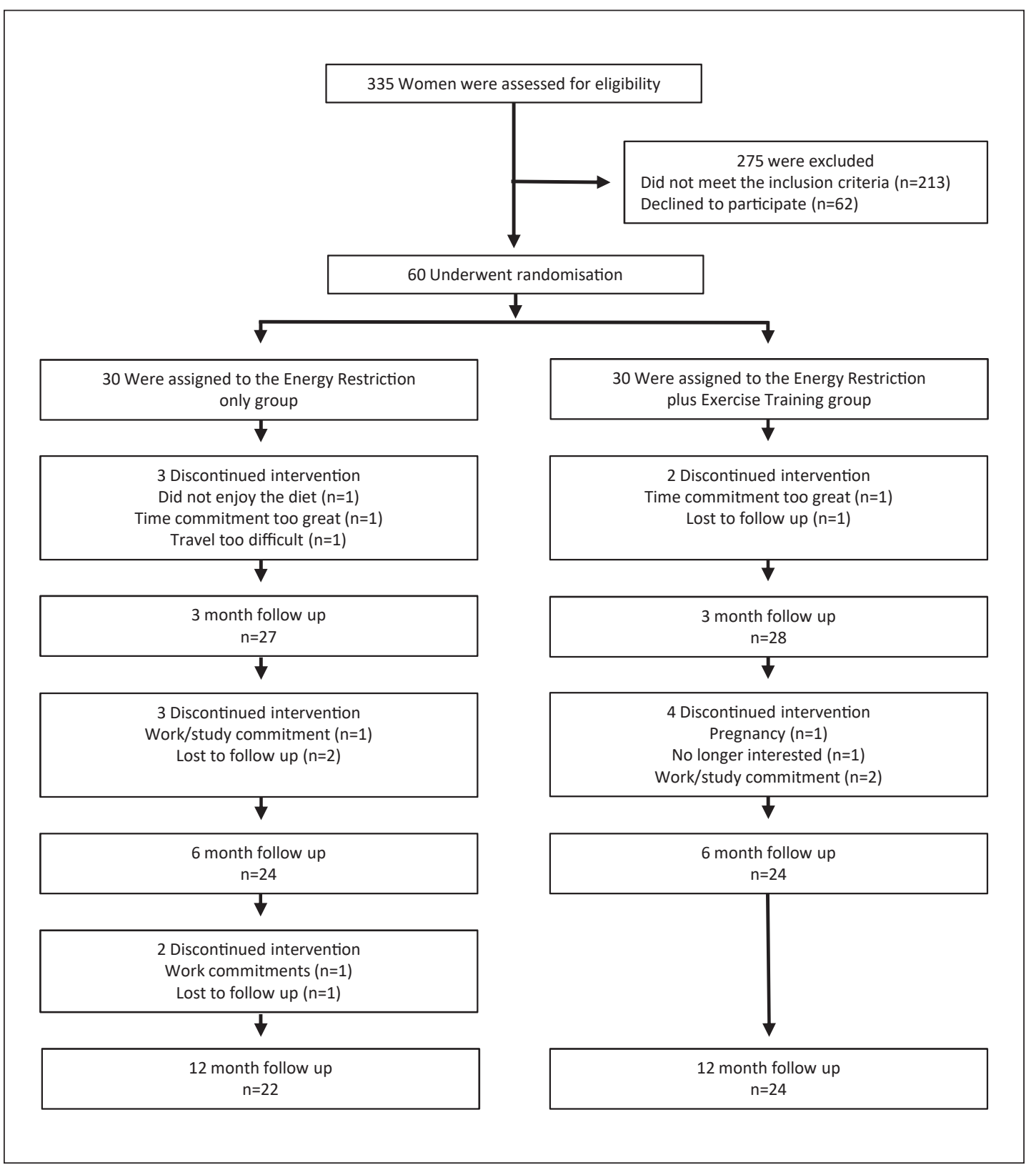

Fig. 1. Participant recruitment, exclusions and a flow chart summarising participant pathways.

and more than 150 min of moderate intensity exercise per week. Baseline data collection was obtained immediately prior to randomisation. Randomisation was performed by an independent researcher external to the project in a block-randomised (blocks of 6) fashion to either energy restriction alone (ER) or energy restriction plus exercise training (EXER) groups using sealed opaque envelopes (online suppl. Appendix S1; for all online suppl. material, see www.karger.com/doi/10.1159/000506643). Participants and researchers involved in delivering participant interventions were not blinded to the group allocation. All participants randomised to ER were informed that they would have the opportunity to attend supervised exercise training at no cost upon study completion for 3 months in an attempt to improve participant retention. 


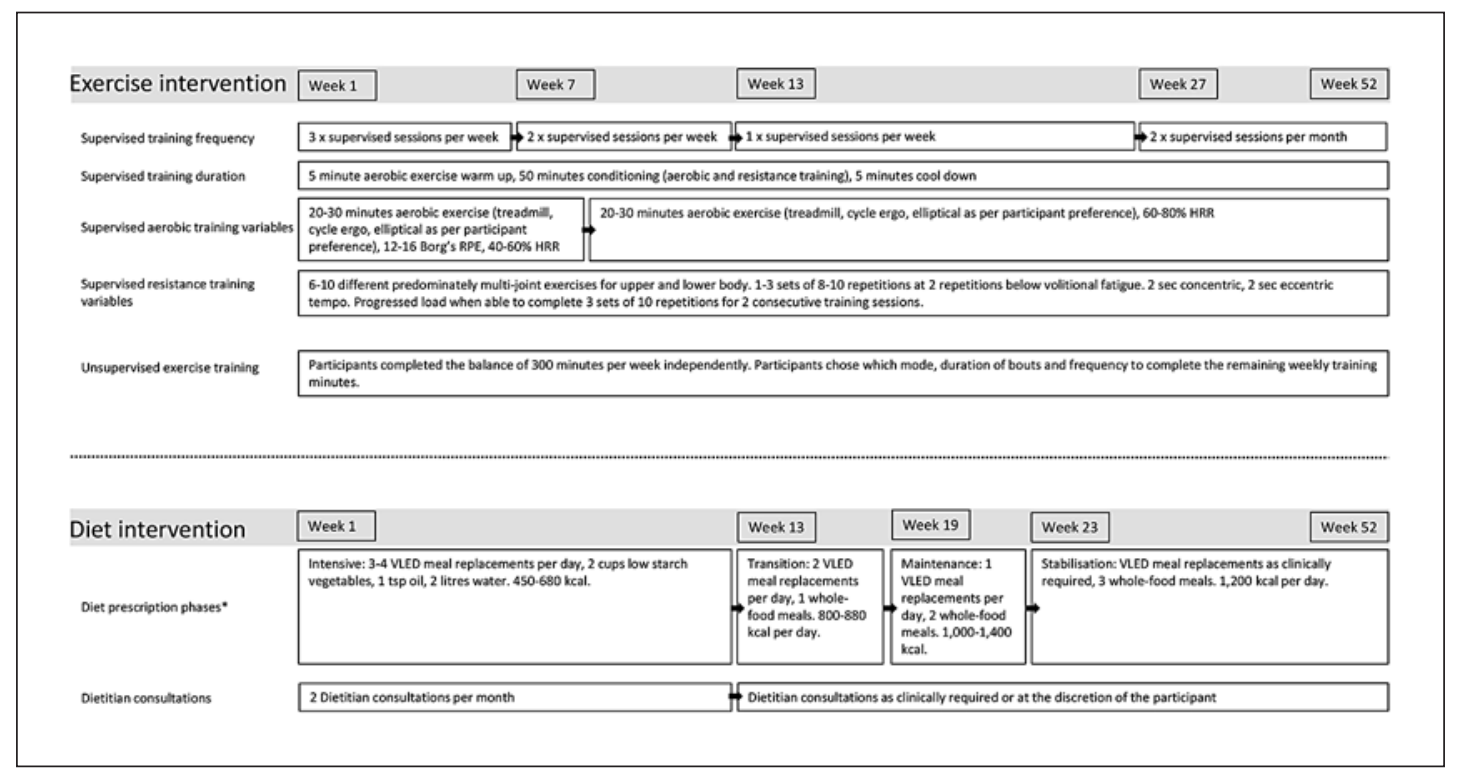

Fig. 2. Overview of the exercise and dietary intervention. VLED, very low energy diet; * Diet prescription was based off the Optifast ${ }^{\circledR}$ clinical treatment protocol. Phase transitions were fluid and determined based on standard clinical practice decision making to facilitate energy deficit or stabilisation of body weight using a client-centred approach.

\section{Energy-Restricted Dietary Intervention}

Both groups received identical energy-restricted diet and dietary consultations (Fig. 2) which were designed to resemble that of standard clinical care for free-living adults by dietitians with experience with severe obesity. The consultations and intervention consisted of a very low energy diet protocol (Optifast ${ }^{\circledR}$, Nestle, Australia) with an energy intake ranging from 450-680 kcal (1,900-2,800 kJ) per day during the intensive phase to 1,200 kcal (5,000 kJ) per day during the stabilisation phase. Participants met every 2 weeks for the first 12 weeks with an accredited practicing dietitian and continued as clinically relevant for the remaining 9 months. All participants started with an intensive phase for at least 12 weeks if tolerated, moving into a transition phase for 6-12 weeks, maintenance phase for the next 6-12 weeks, and then a stabilisation phase for the remaining 6 months. The diet transitioned gradually from full meal replacements to whole food as clinically indicated. There was no attempt to directly control for energy intake beyond the advice and support provided by the study dietitian. The average daily macronutrient content during the intensive phase $(3$ meal replacement products) for the Optifast ${ }^{\circledR}$ diet was: protein $54 \mathrm{~g}$, carbohydrate $59 \mathrm{~g}$, fat $14 \mathrm{~g}$; plus 2 cups of low starch vegetables and 1 teaspoon of oil of the participant's choice. Participants randomised to ER were encouraged to be active but were not provided with specific advice on exercise.

\section{Exercise Training plus Energy-Restricted Diet Intervention}

Participants randomised to EXER completed a supervised exercise training programme (Fig. 2) delivered by accredited exercise physiologists and postgraduate clinical exercise physiology students. Participants underwent a stepped-down approach to supervised exercise training designed to accommodate free-living adults (online suppl. Appendix S1). There was no attempt to control for the degree of energy deficit, energy expenditure or net energy balance between the two interventions. Each supervised exercise training session consisted of 20-30 min aerobic (60-80\% heart rate reserve) and $30 \mathrm{~min}$ of resistance training 
Miller et al.: Exercise Training in Severe Obesity

(1-3 sets of 8-10 repetitions for 8 different upper and lower body exercises) for a total of 60 min. Exercise training consisted of 3 supervised training sessions per week for the first 6 weeks, 2 sessions per week for weeks 7-12, 1 session per week for the next 3 months and 1 session per fortnight for the final 6 months. The supervised training sessions were supplemented with self-administered sessions for a total of $300 \mathrm{~min}$ per week of moderate intensity exercise. The modes of exercise for the self-administered training sessions were developed in consultation with each participant based on their access to facilities and preferences.

\section{Outcome Measures}

Outcome measures were collected at baseline, 3, 6 and 12 months. Participants were instructed to fast overnight and to avoid caffeine for $18 \mathrm{~h}$, heavy exercise and alcohol for $24 \mathrm{~h}$ prior to testing. The co-primary outcome measures were the difference between groups against time at 12 months for peak aerobic power $\left(\mathrm{VO}_{2 \text { peak }}\right)$ expressed in $\mathrm{mL} \cdot \mathrm{kg}^{-1} \cdot \mathrm{min}^{-1}$ and lean body mass in kilograms (dual X-ray absorptiometry).

$\mathrm{VO}_{2 \text { peak }}$ was assessed using a sign- and symptom-limited incremental treadmill test [17]. The speed remained constant throughout the test, and the gradient was increased by $2 \%$ per minute until the participant reached a reported rating of perceived exertion of $17 / 20$ (very hard). Expired respiratory gases were collected and analysed using a breath-by-breath pneumotach system (Innocor Innovision version 6.15, Glamsbjerg, Denmark), and this was calibrated according to the manufacturer guidelines. $\mathrm{HbO}_{2 \text { sat } \%}$ using pulse oximetry was recorded at the end of each minute during the test and recovery.

Three compartment body composition and bone mineral density were assessed using a dual energy X-ray absorptiometry scanner (GE Lunar Prodigy Pro, Madison, WI, USA 53718) with software version 12.1 for total body fat mass, lean body mass, and total body bone mineral content and density. For instances where portions of the trunk remained outside of the scan area, half-body scans were completed. Body weight was measured with participants wearing a hospital gown without shoes. Waist circumference and hip circumference were measured standing using a steel tape measure.

Maximal muscular strength was determined using a 3-repetition maximum for bench press, leg press and seated row using standard resistance training equipment. Full protocol details for strength measures and fasting blood collection and analysis are described in online supplementary Appendix S1 and have been published previously [17].

\section{Statistical Analysis}

All randomised participants were analysed following an intention to treat analysis. Descriptive statistics, i.e. mean and standard deviations (mean \pm SD), were calculated by treatment group for all the variables at baseline.

The change from baseline to each post-baseline time point (mean \pm SEM) was compared between and within the groups using mixed models for repeated measures. This model included fixed effect group, time point (categorical), group and time point interaction, and was adjusted for baseline measurement (centred around mean of each group) by including baseline as a covariate. The model included all individuals who had baseline measurement and at least 1 post-baseline measure and used an unstructured variance-covariance structure to model within-individual errors. There was no formal adjustment for multiple comparisons. Results were reported as mean and standard error unless otherwise specified. All statistical analyses were performed using Stata (StataCorp version 14.2). The sample size for this study was determined based on previous evidence, plus a projected drop-out of $25 \%$, and provided adequate statistical power for the two co-primary outcome measures of $\mathrm{VO}_{2}$ peak $(n=4, \alpha=$ 0.05 , power 0.9 ) [18] and lean body mass $(n=23, S D=1.7, \alpha=0.05$, power 0.8$)$ [19]. A $p$ value of less than 0.05 is considered to be statistically significant. 
Table 1. Baseline characteristics of the participants

\begin{tabular}{|c|c|c|}
\hline Characteristic & Energy restriction & $\begin{array}{l}\text { Energy restriction plus } \\
\text { exercise training }\end{array}$ \\
\hline Age, years & $35.9 \pm 10.3$ & $37.9 \pm 7.69$ \\
\hline Height, m & $1.62 \pm 0.3$ & $1.67 \pm 0.06$ \\
\hline Weight, kg & $114.4 \pm 23.7$ & $111.9 \pm 17.8$ \\
\hline Waist circumference, $\mathrm{cm}$ & $114.3 \pm 13.0$ & $112.5 \pm 11.6$ \\
\hline Hip circumference, $\mathrm{cm}$ & $131.2 \pm 14.3$ & $129.9 \pm 13.7$ \\
\hline Body mass index & $40.6 \pm 6.7$ & $40.2 \pm 6.8$ \\
\hline Fat mass, kg & $59.4 \pm 15.4$ & $56.9 \pm 12.9$ \\
\hline Lean mass, $\mathrm{kg}$ & $50.7 \pm 8.6$ & $50.8 \pm 6.2$ \\
\hline $\mathrm{BMD}, \mathrm{g} / \mathrm{cm}^{3}$ & $1.30 \pm 0.08$ & $1.29 \pm 0.09$ \\
\hline $\mathrm{BMC}, \mathrm{g}$ & $3,184 \pm 372$ & $3,169 \pm 449$ \\
\hline Systolic blood pressure, $\mathrm{mm} \mathrm{Hg}$ & $112.6 \pm 16.6$ & $112.3 \pm 13.5$ \\
\hline Diastolic blood pressure, $\mathrm{mm} \mathrm{Hg}$ & $77.7 \pm 9.9$ & $75.3 \pm 10.2$ \\
\hline Fasting glucose, mmol/L & $5.4 \pm 2$ & $5.2 \pm 0.5$ \\
\hline Total cholesterol, mmol/L & $5.1 \pm 0.7$ & $5.1 \pm 0.8$ \\
\hline Upper body strength, kg & $82.3 \pm 15.5$ & $84.9 \pm 13.4$ \\
\hline Leg strength, kg & $106.9 \pm 22.9$ & $107.7 \pm 19.8$ \\
\hline $\mathrm{VO}_{2 \text { peak, }} \mathrm{mL} \cdot \mathrm{kg}^{-1} \cdot \mathrm{min}^{-1}$ & $25.2 \pm 5.1$ & $26.3 \pm 5.2$ \\
\hline \multicolumn{3}{|l|}{ Chronic diseases $^{\mathrm{a}}, n$} \\
\hline Type 2 diabetes & $1(3.3 \%)$ & 0 \\
\hline Dyslipidaemia & $5(16.7 \%)$ & $3(10 \%)$ \\
\hline Hypertension & $5(16.7 \%)$ & $5(16.7 \%)$ \\
\hline Polycystic ovarian syndrome & $6(20 \%)$ & $5(16.7)$ \\
\hline Depression & $9(30 \%)$ & $8(26.7 \%)$ \\
\hline
\end{tabular}

$\mathrm{BMD}$, bone mineral density; $\mathrm{BMC}$, bone mineral content; $\mathrm{VO}_{2}$ peak, peak aerobic oxygen consumption. Values are means \pm standard deviation, unless otherwise indicated. ${ }^{\text {a }}$ Chronic diseases previously diagnosed and indicated as active medical condition on medical clearance form.

\section{Results}

\section{Study Population}

A total of 60 participants aged $37 \pm 9$ years (mean \pm SD) and a BMI of $40.4 \pm 6.7$ were randomised, and 46 participants (76\%) completed the study (Fig. 1). The characteristics of the two groups are shown in Table 1. All participants with data collected at baseline and at least 1 follow-up time point $(n=53)$ were included in the intention to treat analysis. Attendances at dietary consultations were similar between groups, $12 \pm 5$ (mean \pm SD) dietary consultations in ER and $14 \pm 4$ (mean \pm SD) in EXER (mean difference \pm SEM; $1.9 \pm 1.2 ; p=$ 0.139) (online suppl. Appendix S1). A summary of reported adverse events during study participation are presented in the supplementary material (online suppl. Appendix S1).

\section{Peak Aerobic Power}

$\mathrm{VO}_{2}$ peak relative to total body mass was significantly greater in both groups when compared to baseline at all time points (Table 2). The group difference at 12 months between EXER and ER for $\mathrm{VO}_{2}$ peak relative to total body mass was not statistically significant (mean diff. \pm SEM; $2.3 \pm 1.6 \mathrm{~mL} \cdot \mathrm{kg}^{-1} \bullet \mathrm{min}^{-1}, p=0.15$ ). EXER resulted in significantly greater gains in $\mathrm{VO}_{2 \text { peak }}$ compared to ER at 3 months (mean diff. $\pm \mathrm{SEM} ; 2.5 \pm 0.9 \mathrm{~mL} \cdot \mathrm{kg}^{-1} \cdot \mathrm{min}^{-1}, p=0.006$ ) and 6 months (mean diff. $\pm \mathrm{SEM} ; 3.1 \pm 1.2 \mathrm{~mL} \cdot \mathrm{kg}^{-1} \cdot \mathrm{min}^{-1}, p=0.007$ ) (Table 2).

Absolute $\mathrm{VO}_{2}$ peak $\left(\mathrm{L} \cdot \mathrm{min}^{-1}\right)$ was not significantly different for both EXER and ER over time and there were no significant between-group differences (Table 2). $\mathrm{VO}_{2}$ peak expressed 
Miller et al.: Exercise Training in Severe Obesity

Table 2. The effects of exercise training when added to energy restriction on physical function outcomes

\begin{tabular}{|c|c|c|c|c|}
\hline Outcome & $\begin{array}{l}\text { Energy restriction } \\
\text { only (mean } \pm \text { SEM) }\end{array}$ & $\begin{array}{l}\text { Energy restriction } \\
\text { plus exercise training } \\
(\text { mean } \pm \text { SEM) }\end{array}$ & $\begin{array}{l}\text { Group-time } \\
\text { difference } \\
\text { (mean } \pm \text { SEM) }\end{array}$ & $\begin{array}{l}\text { Group-time } \\
\text { interaction } \\
\text { ( } p \text { value })\end{array}$ \\
\hline \multicolumn{5}{|l|}{$\mathrm{VO}_{2 \text { peak }}, \mathrm{mL} \cdot \mathrm{kg}^{-1} \cdot \mathrm{min}^{-1}$} \\
\hline Baseline & $25.2 \pm 5.1$ & $26.3 \pm 5.2$ & & \\
\hline Change at 3 months & $1.7 \pm 0.7^{*}$ & $4.2 \pm 0.6^{*}$ & $2.5 \pm 0.9$ & 0.006 \\
\hline Change at 6 months & $3.7 \pm 0.8 *$ & $6.7 \pm 0.8^{*}$ & $3.1 \pm 1.2$ & 0.007 \\
\hline Change at 12 months & $3.0 \pm 1.2^{*}$ & $5.4 \pm 1.1^{*}$ & $2.3 \pm 1.6$ & 0.15 \\
\hline \multicolumn{5}{|l|}{ Absolute $\mathrm{VO}_{2}$ peak, $\mathrm{L} \cdot \mathrm{min}^{-1}$} \\
\hline Baseline & $2.82 \pm 0.55$ & $2.86 \pm 0.36$ & & \\
\hline Change at 3 months & $-0.12 \pm 0.07$ & $0.03 \pm 0.06$ & $0.16 \pm 0.09$ & 0.084 \\
\hline Change at 6 months & $0.002 \pm 0.06$ & $0.1 \pm 0.056$ & $0.09 \pm 0.08$ & 0.242 \\
\hline Change at 12 months & $0.007 \pm 0.08$ & $0.11 \pm 0.08$ & $0.1 \pm 0.11$ & 0.352 \\
\hline \multicolumn{5}{|c|}{$\mathrm{VO}_{2 \text { peak }} \mathrm{mL} \cdot \mathrm{kg}^{-1} \cdot \mathrm{min}^{-1}$ lean mass } \\
\hline Baseline & $56.0 \pm 9.7$ & $56.9 \pm 8.3$ & & \\
\hline Change at 3 months & $1.59 \pm 1.56$ & $4.23 \pm 1.51^{*}$ & $2.63 \pm 2.19$ & 0.231 \\
\hline Change at 6 months & $2.53 \pm 1.47$ & $4.84 \pm 1.39 *$ & $2.31 \pm 2.02$ & 0.252 \\
\hline Change at 12 months & $2.57 \pm 1.68$ & $2.63 \pm 2.19$ & $1.25 \pm 2.29$ & 0.584 \\
\hline \multicolumn{5}{|l|}{ Upper body strength, kg } \\
\hline Baseline & $82.3 \pm 15.5$ & $84.9 \pm 13.4$ & & \\
\hline Change at 3 months & $-4.3 \pm 1.2^{*}$ & $1.4 \pm 1.2$ & $5.6 \pm 1.7$ & 0.001 \\
\hline Change at 6 months & $-2.7 \pm 1.4$ & $3.9 \pm 1.5^{*}$ & $6.6 \pm 2.0$ & 0.001 \\
\hline Change at 12 months & $-1.3 \pm 1.5$ & $7.3 \pm 1.5^{*}$ & $8.6 \pm 2.0$ & $<0.001$ \\
\hline \multicolumn{5}{|l|}{ Leg press strength, kg } \\
\hline Baseline & $106.9 \pm 22.9$ & $107.7 \pm 19.8$ & & \\
\hline Change at 3 months & $0.9 \pm 3.7$ & $12.8 \pm 3.6^{*}$ & $11.8 \pm 5.2$ & 0.021 \\
\hline Change at 6 months & $5.5 \pm 4.3$ & $24.7 \pm 4.3^{*}$ & $19.9 \pm 6.0$ & 0.001 \\
\hline Change at 12 months & $9.1 \pm 5.5$ & $28.3 \pm 5.3^{*}$ & $19.3 \pm 7.6$ & 0.011 \\
\hline \multicolumn{5}{|l|}{ Total strength, kg } \\
\hline Baseline & $189.2 \pm 33.5$ & $192.0 \pm 29.4$ & & \\
\hline Change at 3 months & $-2.9 \pm 4.0$ & $15.0 \pm 4.0^{*}$ & $17.9 \pm 5.7$ & 0.002 \\
\hline Change at 6 months & $2.9 \pm 4.5$ & $30.5 \pm 4.6^{*}$ & $27.7 \pm 6.5$ & $<0.001$ \\
\hline Change at 12 months & $7.1 \pm 6.0$ & $36.5 \pm 5.6^{*}$ & $29.4 \pm 8.2$ & $<0.001$ \\
\hline
\end{tabular}

${ }^{*} p<0.05$ : statistical significance within-group change from baseline. SEM, standard error of the mean. Baseline presented as mean \pm SD.

relative to lean mass increased above baseline for EXER at 3 months $\left(4.23 \pm 1.51 \mathrm{~mL} \cdot \mathrm{kg}^{-1}\right.$. $\left.\min ^{-1} ; p<0.05\right)$ and 6 months $\left(4.84 \pm 1.39 \mathrm{~mL} \cdot \mathrm{kg}^{-1} \cdot \mathrm{min}^{-1}, p<0.05\right)$, but not at 12 months. There were no changes for ER at any time point, and there were no between-group effects at any time point (Table 2).

\section{Body Composition}

The addition of exercise training to energy restriction facilitated body mass loss at 3 months (EXER vs. ER) but not at 6 or 12 months (Table 3). Lean body mass was similarly reduced at all time points compared to baseline for both groups, and there was no difference between EXER and ER at 12 months for lean body mass $(0.3 \pm 1.0 \mathrm{~kg}, p=0.352$; Table 3$)$. Both EXER and ER exhibited significant reductions in total body mass at all time points compared to baseline (Table 3). A 5\% body mass loss or greater was achieved by $60 \%$ of participants in ER, and $82 \%$ in EXER at 12 months (Fig. 3). Fat mass was reduced to a greater extent in EXER at 3 months $(p<0.001)$, trended to be greater at 6 months $(p=0.062)$ and was not significantly different at 12 months when compared to ER (Table 3). At 12 months, the ratio of total 
Miller et al.: Exercise Training in Severe Obesity

Table 3. The effects of exercise training when added to energy restriction on anthropometric outcomes

\begin{tabular}{|c|c|c|c|c|}
\hline Outcome & $\begin{array}{l}\text { Energy } \\
\text { restriction only } \\
\text { (mean } \pm \text { SEM) }\end{array}$ & $\begin{array}{l}\text { Energy restriction } \\
\text { plus exercise training } \\
(\text { mean } \pm \text { SEM) }\end{array}$ & $\begin{array}{l}\text { Group-time } \\
\text { difference } \\
(\text { mean } \pm \text { SEM) }\end{array}$ & $\begin{array}{l}\text { Group-time } \\
\text { interaction } \\
(p \text { value })\end{array}$ \\
\hline \multicolumn{5}{|l|}{ Weight, kg } \\
\hline Baseline & $114.1 \pm 23.6$ & $111.4 \pm 17.5$ & & \\
\hline Change at 3 months & $-9.9 \pm 1.3^{*}$ & $-14.6 \pm 1.2^{*}$ & $4.7 \pm 1.75$ & 0.006 \\
\hline Change at 6 months & $-10.9 \pm 2.2^{*}$ & $-16.1 \pm 2.2^{*}$ & $5.2 \pm 3.1$ & 0.093 \\
\hline Change at 12 months & $-8.9 \pm 2.7^{*}$ & $-12.3 \pm 2.7^{*}$ & $3.4 \pm 3.8$ & 0.368 \\
\hline \multicolumn{5}{|l|}{ Body mass index } \\
\hline Baseline & $40.6 \pm 6.7$ & $40.2 \pm 6.8$ & & \\
\hline Change at 3 months & $-3.55 \pm 0.5^{*}$ & $-5.3 \pm 0.4^{*}$ & $1.73 \pm 0.6$ & 0.006 \\
\hline Change at 6 months & $-3.9 \pm 0.8^{*}$ & $-5.75 \pm 0.8^{*}$ & $1.84 \pm 1.1$ & 0.101 \\
\hline Change at 12 months & $-3.3 \pm 1.0^{*}$ & $-4.45 \pm 1.0^{*}$ & $1.19 \pm 1.4$ & 0.39 \\
\hline \multicolumn{5}{|l|}{ Fat mass, kg } \\
\hline Baseline & $59.4 \pm 15.4$ & $56.9 \pm 12.9$ & & \\
\hline Change at 3 months & $-6.5 \pm 1.0^{*}$ & $-11.4 \pm 1.0^{*}$ & $4.9 \pm 1.9$ & $<0.001$ \\
\hline Change at 6 months & $-9.3 \pm 2.0 *$ & $-14.5 \pm 1.9 *$ & $5.2 \pm 2.8$ & 0.062 \\
\hline Change at 12 months & $-8.1 \pm 2.8^{*}$ & $-11.2 \pm 2.7^{*}$ & $3.1 \pm 3.9$ & 0.420 \\
\hline \multicolumn{5}{|l|}{ Lean mass, $\mathrm{kg}$} \\
\hline Baseline & $50.7 \pm 8.6$ & $50.8 \pm 6.2$ & & \\
\hline Change at 3 months & $-3.4 \pm 0.7^{*}$ & $-3.0 \pm 0.6^{*}$ & $0.4 \pm 0.9$ & 0.621 \\
\hline Change at 6 months & $-1.9 \pm 0.7^{*}$ & $-2.2 \pm 0.7^{*}$ & $0.3 \pm 1.0$ & 0.785 \\
\hline Change at 12 months & $-1.7 \pm 0.8^{*}$ & $-1.4 \pm 0.7^{*}$ & $0.3 \pm 1.0$ & 0.777 \\
\hline \multicolumn{5}{|l|}{ Waist circumference, $\mathrm{cm}$} \\
\hline Baseline & $114.3 \pm 13.0$ & $112.7 \pm 11.6$ & & \\
\hline Change at 3 months & $-7.5 \pm 1.3^{*}$ & $-12.3 \pm 1.3^{*}$ & $4.8 \pm 1.9$ & 0.009 \\
\hline Change at 6 months & $-9.6 \pm 1.9 *$ & $-13.5 \pm 1.9 *$ & $3.9 \pm 2.7$ & 0.144 \\
\hline Change at 12 months & $-9.1 \pm 2.2^{*}$ & $-12.6 \pm 2.1^{*}$ & $3.5 \pm 3.1$ & 0.257 \\
\hline \multicolumn{5}{|l|}{ Hip circumference, $\mathrm{cm}$} \\
\hline Baseline & $130.2 \pm 14.3$ & $129.9 \pm 13.7$ & & \\
\hline Change at 3 months & $-6.8 \pm 1.2^{*}$ & $-11.3 \pm 1.2^{*}$ & $4.5 \pm 1.7$ & 0.007 \\
\hline Change at 6 months & $-7.0 \pm 1.9^{*}$ & $-13.2 \pm 1.9^{*}$ & $6.2 \pm 2.7$ & 0.023 \\
\hline Change at 12 months & $-5.9 \pm 2.5^{*}$ & $-9.6 \pm 2.4^{*}$ & $3.6 \pm 3.4$ & 0.289 \\
\hline
\end{tabular}

$* p<0.05$ : statistical significance within-group change from baseline. SEM, standard error of the mean. Baseline presented as mean \pm standard deviation.

mass loss to lean body mass loss was 5.6:1 kg in ER, and 9.3:1 kg in EXER. Expressed as percentages, $17.9 \%$ of total mass loss in ER was comprised of lean body mass, and $10.8 \%$ in EXER at 12 months.

\section{Muscle Strength}

Total muscle strength (calculated as the sum of weight lifted for all three strength tests) increased significantly in EXER but not in ER at 3 months (mean \pm SEM; $15.0 \pm 4.0 \mathrm{~kg}, p<$ $0.001,-2.88 \pm 4.0 \mathrm{~kg}$, respectively; $p=0.476$ ), 6 months (mean $\pm \mathrm{SEM} ; 30.5 \pm 4.6 \mathrm{~kg}, p<0.001$, $2.8 \pm 4.5 \mathrm{~kg}$, respectively; $p=0.530$ ) and 12 months (mean $\pm \mathrm{SEM} ; 36.5 \pm 5.6 \mathrm{~kg}, p<0.001$, $7.1 \pm 6.0 \mathrm{~kg}$, respectively; $p=0.242$ ) (Table 2).

\section{Cardiovascular Risk Factors}

No differences between groups at 12 months were observed for total cholesterol, lowdensity lipoprotein cholesterol, high-density lipoprotein cholesterol, triglycerides, systolic or diastolic blood pressure or fasting plasma glucose (Table 4). At 12 months, within-group 


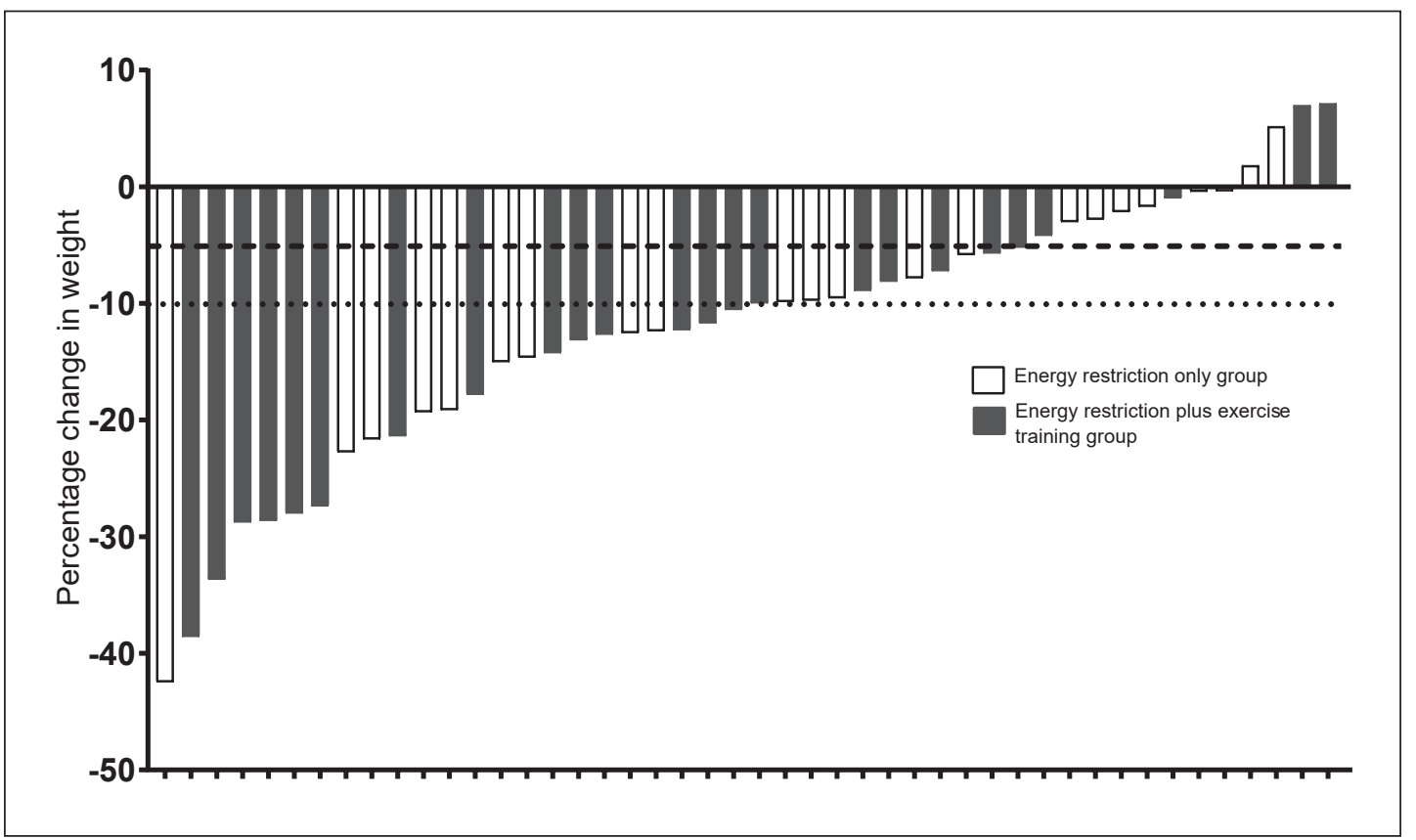

Fig. 3. Individual percentage weight change between baseline and 12 months for energy restriction and energy restriction plus exercise training. Each bar represents a single participant for their proportion of weight change from baseline to 12 months. The dashed line represents a $5 \%$ weight loss at 12 months. The dotted line represents $10 \%$ weight loss at 12 months.

improvements for high-density lipoprotein cholesterol were observed for both groups. Additionally, ER showed improved fasting glucose while EXER did not. Improvements for fasting glucose occurred in both groups at 3 and 6 months but were maintained in ER (mean $-0.18 \pm$ $0.07, p<0.05$ ) and not EXER (mean $-0.06 \pm 0.07, p>0.05$ ) at 12 months. Systolic and diastolic blood pressure improved in both groups at 3 and 6 months but not at 12 months. There were no significant differences between groups at any time point for systolic or diastolic blood pressure.

\section{Discussion}

The addition of exercise training to an energy-restricted diet in women with severe obesity did not lead to greater changes in aerobic power or preservation of lean mass at 12 months, compared to energy restriction alone. Both groups experienced significant improvements in aerobic power and a modest, yet statistically significant loss of lean mass at the trial end.

Few prolonged studies such as this 12-month trial have evaluated the effect of exercise training when added to energy restriction in premenopausal women with clinically severe obesity for changes in lean mass. One 12-month study [20] showed that exercise training added to diet limited the loss of lean mass; however, this was in older adults and may not be generalisable to younger cohorts. Preservation of lean mass for shorter duration interventions with younger adults with obesity class I [21], class II [22] and class III [23-25] showed mixed results. The addition of exercise training to surgically induced weight loss in severe obesity may positively influence lean mass [26]; however, randomised controlled trials also showed mixed results. The addition of moderate intensity aerobic exercise training [27], 
Miller et al.: Exercise Training in Severe Obesity

Table 4. The effects of exercise training when added to energy restriction on markers of cardiometabolic risk

\begin{tabular}{|c|c|c|c|c|}
\hline Outcome & $\begin{array}{l}\text { Energy } \\
\text { restriction only } \\
(\text { mean } \pm \text { SEM) }\end{array}$ & $\begin{array}{l}\text { Energy restriction } \\
\text { plus exercise training } \\
\text { (mean } \pm \text { SEM) }\end{array}$ & $\begin{array}{l}\text { Group-time } \\
\text { difference } \\
(\text { mean } \pm \text { SEM) }\end{array}$ & $\begin{array}{l}\text { Group-time } \\
\text { interaction } \\
\text { ( } p \text { value })\end{array}$ \\
\hline \multicolumn{5}{|l|}{ Total cholesterol, mmol/L } \\
\hline Baseline & $5.10 \pm 0.13$ & $5.10 \pm 0.15$ & & \\
\hline Change at 3 months & $-0.16 \pm 0.14$ & $-0.57 \pm 0.13^{*}$ & $0.41 \pm 0.19$ & 0.034 \\
\hline Change at 6 months & $-0.1 \pm 0.16$ & $-0.26 \pm 0.16$ & $0.16 \pm 0.23$ & 0.489 \\
\hline Change at 12 months & $0.05 \pm 0.20$ & $-0.26 \pm 0.18$ & $0.31 \pm 0.27$ & 0.256 \\
\hline \multicolumn{5}{|l|}{ LDL cholesterol, mmol/L } \\
\hline Baseline & $3.04 \pm 0.12$ & $3.11 \pm 0.12$ & & \\
\hline Change at 3 months & $0.00 \pm 0.12$ & $-0.33 \pm 0.11^{*}$ & $0.34 \pm 0.16$ & 0.038 \\
\hline Change at 6 months & $-0.02 \pm 0.14$ & $-0.24 \pm 0.14$ & $0.22 \pm 0.20$ & 0.273 \\
\hline Change at 12 months & $-0.37 \pm 0.87$ & $1.04 \pm 0.76$ & $1.41 \pm 1.16$ & 0.220 \\
\hline \multicolumn{5}{|l|}{ HDL cholesterol, mmol/L } \\
\hline Baseline & $1.46 \pm 0.06$ & $1.35 \pm 0.05$ & & \\
\hline Change at 3 months & $-0.09 \pm 0.05^{*}$ & $-0.04 \pm 0.04$ & $0.05 \pm 0.06$ & 0.450 \\
\hline Change at 6 months & $0.027 \pm 0.05$ & $0.11 \pm 0.05^{*}$ & $0.09 \pm 0.07$ & 0.206 \\
\hline Change at 12 months & $0.18 \pm 0.09 *$ & $0.31 \pm 0.08^{*}$ & $0.13 \pm 0.12$ & 0.277 \\
\hline \multicolumn{5}{|l|}{ Triglycerides, mmol/L } \\
\hline Baseline & $1.18 \pm 0.07$ & $1.32 \pm 0.11$ & & \\
\hline Change at 3 months & $-0.17 \pm 0.07^{*}$ & $-0.43 \pm 0.06^{*}$ & $0.26 \pm 0.09$ & 0.003 \\
\hline Change at 6 months & $-0.24 \pm 0.08^{*}$ & $-0.33 \pm 0.08^{*}$ & $0.09 \pm 0.11$ & 0.440 \\
\hline Change at 12 months & $-0.18 \pm 0.11$ & $-0.16 \pm 0.10$ & $0.02 \pm 0.15$ & 0.907 \\
\hline \multicolumn{5}{|c|}{ Systolic blood pressure, $\mathrm{mm} \mathrm{Hg}$} \\
\hline Baseline & $112.6 \pm 3.0$ & $112.3 \pm 2.5$ & & \\
\hline Change at 3 months & $-5.4 \pm 1.7^{*}$ & $-8.0 \pm 1.7^{*}$ & $2.7 \pm 2.4$ & 0.275 \\
\hline Change at 6 months & $-4.9 \pm 1.7^{*}$ & $-5.7 \pm 1.7^{*}$ & $0.9 \pm 2.4$ & 0.720 \\
\hline Change at 12 months & $0.1 \pm 2.1$ & $-3.1 \pm 2.0$ & $3.1 \pm 2.9$ & 0.285 \\
\hline \multicolumn{5}{|c|}{ Diastolic blood pressure, $\mathrm{mm} \mathrm{Hg}$} \\
\hline Baseline & $77.7 \pm 1.8$ & $75.3 \pm 1.9$ & & \\
\hline Change at 3 months & $-5.4 \pm 1.3^{*}$ & $-8.5 \pm 1.3^{*}$ & $3.2 \pm 1.8$ & 0.084 \\
\hline Change at 6 months & $-7.4 \pm 1.5^{*}$ & $-6.6 \pm 1.5^{*}$ & $0.8 \pm 2.1$ & 0.692 \\
\hline Change at 12 months & $-3.1 \pm 1.8$ & $-2.9 \pm 1.8$ & $0.2 \pm 2.5$ & 0.952 \\
\hline \multicolumn{5}{|l|}{ Fasting glucose, $\mathrm{mmol} / \mathrm{L}$} \\
\hline Baseline & $5.4 \pm 0.37$ & $5.2 \pm 0.10$ & & \\
\hline Change at 3 months & $-0.23 \pm 0.07^{*}$ & $-0.19 \pm 0.06^{*}$ & $0.05 \pm 0.09$ & 0.614 \\
\hline Change at 6 months & $-0.19 \pm 0.08^{*}$ & $-0.25 \pm 0.08^{*}$ & $0.06 \pm 0.11$ & 0.621 \\
\hline Change at 12 months & $-0.18 \pm 0.07^{*}$ & $-0.06 \pm 0.07$ & $0.11 \pm 0.10$ & 0.249 \\
\hline
\end{tabular}

${ }^{*} p<0.05$ : statistical significance within-group change from baseline. LDL, low-density lipoprotein; HDL, highdensity lipoprotein; SEM, standard error of the mean. Baseline presented as mean \pm standard deviation.

aerobic plus resistance training [28] or resistance training alone $[29,30]$ over 12 weeks does not lead to differences in lean mass when compared to standard care control groups in participants after Roux-en-Y gastric bypass surgery. There is only one study of 12 months duration which showed that combined aerobic and resistance training after Roux-en-Y gastric bypass protected against lean mass loss when compared to the nonexercise surgery group [31]. However, this was not a randomised clinical trial and the method of group allocation was not described.

While there is evidence that a loss of lean mass occurs concurrently with weight loss in the order of approximately $30 \%$ of total weight loss for women with class II obesity [32], we showed that weight loss with and without a structured exercise programme resulted in lower than predicted relative lean mass loss (EXER, 10.8\%; ER, 17.9\%). The addition of exercise 
Miller et al.: Exercise Training in Severe Obesity

training in our study did not result in a regain of lean mass as reported in a shorter 12-week study of men and women [23]. However, that study implemented a higher-intensity resistance training protocol in the absence of aerobic training, was of shorter duration and differed with respect to participant characteristics. Although various studies have explored the effect of exercise training during weight loss in adults with obesity, there remains insufficient evidence to confirm the ability for protection of lean mass during hypocaloric diets or bariatric surgery in adults with clinically severe obesity. Our data confirm that the addition of a combined aerobic and resistance training programme to energy restriction did not lead to protection of lean body mass when compared to energy restriction alone in free-living women with clinically severe obesity. However, the supervised resistance training intervention in our study also reduced in frequency to twice per month in the last 6 months of the trial. This volume of training is not likely sufficient to observe a gain in lean mass unless participants chose to complete resistance exercise outside of supervised sessions. In the absence of such differences between groups for lean body mass, total-body strength increased in EXER when compared to ER at 12 months. These results are consistent with previous evidence to show that resistance training increases strength and skeletal muscle cross-sectional area in the absence of lean mass gain during a hypocaloric diet [33], which may assist in the protection of future physical disability and early mortality [34].

Peak aerobic fitness is an important determinant of physical function and future physical disability [35], and a strong predictor of cardiovascular events and early mortality [36]. We showed that both ER and EXER enhanced aerobic power relative to body weight at 12 months by 3 and $5.4 \mathrm{~mL} \cdot \mathrm{kg}^{-1} \cdot \mathrm{min}^{-1}$, respectively, but there was no significant difference between groups at the study end. This is in contrast to a previous meta-analysis of long duration diet and exercise studies in adults with overweight and obesity, where combined exercise training with an energy-restricted diet led to greater aerobic fitness benefits when compared to energy restriction alone [37], but none of the studies included in that review reported on aerobic fitness in premenopausal women with class II obesity. To our knowledge, no longterm studies have evaluated the effects of combined exercise training and energy restriction in premenopausal women with clinically severe obesity for changes in aerobic fitness. One study of premenopausal women with class I obesity followed over 12 months showed aerobic exercise training during an energy-restricted diet led to greater aerobic power than diet only [38]. This study consisted of a similar aerobic training prescription as our study; however, supervised training continued 3 times per week for 12 months, possibly influencing adherence to the intended training stimulus and therefore the results [39]. This explanation may support our observations at 3 and 6 months where aerobic power increased more in EXER when compared to ER when supervision was greater. Encouragingly, both groups demonstrated improved aerobic power relative to body weight; however, further evidence is required to determine long-term effective exercise prescription approaches which may be suitable for premenopausal women with clinically severe obesity.

Weight loss and specifically fat mass loss are important for the reduction of cardiovascular disease risk and all-cause mortality [40]. Previous evidence from long-term studies shows that exercise when added to diet provides a modest additional loss of body mass and fat mass in adults with overweight and obesity [37]. Our results show that exercise when added to an energy-restricted diet did not reduce fat mass or weight more when compared to the energy-restricted diet-only group at 12 months for women with clinically severe obesity. Similar studies in adults following bariatric surgery confirm a modest additive effect of exercise [41]; however, the only study in women completing aerobic plus resistance training after bariatric surgery showed there was no additional fat mass loss at 24 months when compared to the non-intervention surgery-only group [42]. The lack of additional weight loss and fat mass loss beyond the 3-month follow-up may be associated with a non- 
Miller et al.: Exercise Training in Severe Obesity

linear weight loss trajectory observed in previous weight loss research [8], or the steppeddown approach to exercise supervision. However, the study design does not allow for confirmation of the reason for the lack of difference between groups at 12 months. There continues to be a lack of research in this population group and inconsistent results further support the need for additional research in this area.

Traditional markers of cardiometabolic risk remained relatively unchanged from baseline to the follow-up at 12 months. The non-significant changes in systolic and diastolic blood pressure in both groups likely reflects that participants were normotensive at baseline. Previous evidence suggests that a similar weight loss to that achieved in this study leads to a 7- to 10-mm $\mathrm{Hg}$ reduction in systolic blood pressure and a 7-mm Hg reduction in diastolic blood pressure [43]; however, this was not the case in these normotensive women with severe obesity. Similar improvements at 12 months were observed in both groups at 12 months for total cholesterol, low-density lipoprotein cholesterol and triglycerides when compared to baseline. Both groups demonstrated improved high-density lipoprotein cholesterol at 12 months when compared to baseline, while only the ER group demonstrated reduced fasting plasma glucose. It is well accepted that lifestyle interventions which result in $5 \%$ or more weight loss using diet, exercise or combined treatments [44] lead to improved biochemical markers of cardiometabolic disease risk in adults with obesity and existing metabolic impairments. The absence of improved cardiometabolic markers in this cohort is likely due to the relatively normal baseline findings.

Based on the results presented here, it appears that premenopausal women with clinically severe obesity do not achieve greater weight loss, fat mass loss or improve aerobic power more when prescribed an aerobic plus resistance training programme with energy restriction over an energy-restricted diet alone under free-living conditions. These findings are important because little is known about how premenopausal women with clinically severe obesity respond to a diet and exercise intervention in free-living conditions for measures of body composition and physical fitness. Although the results presented here showed little additional benefit outside of strength improvement at 12 months with the addition of exercise training, it is reasonable to expect that an exercise intervention that strengthens the support for these women more than in our trial may be effective in enhancing body composition and fitness beyond 6 months in premenopausal women with clinically severe obesity. Based on the superior results at 3 months for the majority of the outcomes in EXER when compared with $E R$, it is important that further research evaluates the effect of supervision models which retain intensive diet and exercise interventions over a longer period of follow-up.

\section{Strengths and Limitations}

Strengths included the prolonged period of follow-up, the method used to evaluate body composition, the recruitment of women with clinically severe obesity and the ecologically valid study design. Limitations included: (i) the reasonably high level of participant attrition that compromised our anticipated statistical power for the primary end points at 12 months; (ii) participants only achieved slightly more than half of the intended exercise training at all time points (see online suppl. Appendix S1). This may bring into question whether $300 \mathrm{~min}$ of weekly exercise is feasible for women with clinically severe obesity, despite the recommendations that 200-300 min per week is necessary to promote long-term weight loss [45]; (iii) the groups were not matched for the degree of energy deficit, which makes conclusions about the actual effect of exercise in addition to diet difficult. However, this was not the intention of the study design. This study was developed to compare the effect of the two interventions, in free-living conditions under the guidance of experienced allied health professionals in a community setting. This is an important distinction to make given the real-world applicability the findings from this study may provide for clinical practice. 
Miller et al.: Exercise Training in Severe Obesity

\section{Conclusion}

Combining exercise training with an energy-restricted diet did not lead to greater aerobic power or improvements in lean body mass, fat mass or total body mass at 12 months when compared to energy restriction alone for premenopausal women with clinically severe obesity in free-living situations. Further research is required to determine an effective and feasible exercise and diet approach which can be applied in the community setting for these high-risk individuals.

\section{Acknowledgements}

The authors would like to thank the YMCA Victoria, Australia, for its support of this trial, offering in-kind access to its gymnasium facilities at Kew Recreation Centre, RecWest Footscray, Ascot Vale Leisure Centre, Knox Leisureworks, Burwood Fitness Centre and Endeavour Hills Leisure Centre.

The authors would also like to acknowledge the participants who volunteered their time to support this study. The full study protocol has been published previously [17]. Individual participant data will not be made available or shared outside of the research group.

\section{Statement of Ethics}

The study was approved by the Human Research Ethics Committees of the Alfred Hospital (HREC reference 2011-154) and Deakin University (HREC reference 330-11) in accordance with the guidelines of the National Health and Medical Research Council of Australia (Australian New Zealand Clinical Trials Registry, ACTRN12611000694910).

\section{Disclosure Statement}

John B. Dixon is a board member of Nestlé Health Sciences Australia and has received payment for consultancy from Apollo Endosurgery, Bariatric Advantage, iNova Pharmaceuticals and Novo Nordisk. The laboratories of Professors Dixon and Lambert have recently received research funding from Medtronic. Gavin Lambert has received payment for consultancy from Medtronic. Clint T. Miller, Steve F. Fraser, Steve E. Selig, Toni Rice, Mariee Grima, Daniel J. van den Hoek and Carolina Ika Sari declare no conflicts of interest.

\section{Funding Statement}

Nestlé Health Australia provided VLED products at cost in support of the study. John B. Dixon and Gavin W. Lambert were supported by Senior Research Fellowships of the Australian National Health and Medical Research Council. While this study has not received any other external funding, work at the various institutions was supported in part by the Victorian Government's Operational Infrastructure Support Program. 


\section{Author Contributions}

The authors declare substantial contribution by all authors to the development of the study design, acquisition of data, analysis and preparation of the manuscript.

\section{References}

1 NCD Risk Factor Collaboration. Trends in adult body-mass index in 200 countries from 1975 to 2014: a pooled analysis of 1,698 population-based measurement studies with 19.2 million participants. The Lancet. 387(10026):1377-96.

2 Dixon JB. The effect of obesity on health outcomes. Mol Cell Endocrinol. 2010 Mar;316(2):104-8.

3 Andreyeva T, Sturm R, Ringel JS. Moderate and severe obesity have large differences in health care costs. Obes Res. 2004 Dec;12(12):1936-43.

4 Jebb SA, Moore MS. Contribution of a sedentary lifestyle and inactivity to the etiology of overweight and obesity: current evidence and research issues. Med Sci Sports Exerc. 1999 Nov;31(11 Suppl):S534-41.

5 He XZ, Baker DW. Body mass index, physical activity, and the risk of decline in overall health and physical functioning in late middle age. Am J Public Health. 2004 Sep;94(9):1567-73.

6 Stenholm S, Strandberg TE, Pitkala K, Sainio P, Heliovaara M, Koskinen S. Midlife obesity and risk of frailty in old age during a 22-year follow-up in men and women: the Mini-Finland Follow-Up Survey. J Gerontol A Biol Sci Med Sci. 2014 Jan;69(1):73-8.

7 Council NHMR, editor. Clinical practice guidelines for the management of overweight and obesity in adults, adolescents and children in Australia - systematic review. Canberra: Commonwealth of Australia; 2013.

8 Franz MJ, VanWormer JJ, Crain AL, Boucher JL, Histon T, Caplan W, et al. Weight-loss outcomes: a systematic review and meta-analysis of weight-loss clinical trials with a minimum 1-year follow-up. J Am Diet Assoc. 2007 Oct;107(10):1755-67.

9 Wu T, Gao X, Chen M, van Dam RM. Long-term effectiveness of diet-plus-exercise interventions vs. diet-only interventions for weight loss: a meta-analysis. Obes Rev. 2009 May;10(3):313-23.

10 Miller CT, Fraser SF, Levinger I, Straznicky NE, Dixon JB, Reynolds J, et al. The effects of exercise training in addition to energy restriction on functional capacities and body composition in obese adults during weight loss: a systematic review. PLoS One. 2013 Nov;8(11):e81692.

11 Pescatello LS, Kelsey BK, Price TB, Seip RL, Angelopoulos TJ, Clarkson PM, et al. The muscle strength and size response to upper arm, unilateral resistance training among adults who are overweight and obese. J Strength Cond Res. 2007 May;21(2):307-13.

12 Guillet C, Masgrau A, Walrand S, Boirie Y. Impaired protein metabolism: interlinks between obesity, insulin resistance and inflammation. Obes Rev. 2012 Dec;13 Suppl 2:51-7.

13 Straznicky NE, Lambert GW, Masuo K, Dawood T, Eikelis N, Nestel PJ, et al. Blunted sympathetic neural response to oral glucose in obese subjects with the insulin-resistant metabolic syndrome. Am J Clin Nutr. 2009 Jan;89(1):27-36.

14 Carey AL, Formosa MF, Van Every B, Bertovic D, Eikelis N, Lambert GW, et al. Ephedrine activates brown adipose tissue in lean but not obese humans. Diabetologia. 2013 Jan;56(1):147-55.

15 Toledo FG, Goodpaster BH. The role of weight loss and exercise in correcting skeletal muscle mitochondrial abnormalities in obesity, diabetes and aging. Mol Cell Endocrinol. 2013 Oct;379(1-2):30-4.

16 Garber CE, Blissmer B, Deschenes MR, Franklin BA, Lamonte MJ, Lee IM, et al.; American College of Sports Medicine. American College of Sports Medicine position stand. Quantity and quality of exercise for developing and maintaining cardiorespiratory, musculoskeletal, and neuromotor fitness in apparently healthy adults: guidance for prescribing exercise. Med Sci Sports Exerc. 2011 Jul;43(7):1334-59.

17 Miller CT, Fraser SF, Selig SE, Rice T, Grima M, Straznicky NE, et al. The functional and clinical outcomes of exercise training following a very low energy diet for severely obese women: study protocol for a randomised controlled trial. Trials. 2016 Mar;17(1):125.

18 Straznicky NE, Lambert EA, Grima MT, Eikelis N, Nestel PJ, Dawood T, et al. The effects of dietary weight loss with or without exercise training on liver enzymes in obese metabolic syndrome subjects. Diabetes Obes Metab. 2012 Feb;14(2):139-48.

19 Straznicky NE, Grima MT, Lambert EA, Eikelis N, Dawood T, Lambert GW, et al. Exercise augments weight loss induced improvement in renal function in obese metabolic syndrome individuals. J Hypertens. 2011 Mar; 29(3):553-64.

20 Villareal DT, Chode S, Parimi N, Sinacore DR, Hilton T, Armamento-Villareal R, et al. Weight loss, exercise, or both and physical function in obese older adults. N Engl J Med. 2011 Mar;364(13):1218-29.

21 Chaston TB, Dixon JB, O'Brien PE. Changes in fat-free mass during significant weight loss: a systematic review. Int J Obes. 2007 May;31(5):743-50.

22 Freitas PD, Ferreira PG, Silva AG, Stelmach R, Carvalho-Pinto RM, Fernandes FL, et al. The Role of Exercise in a Weight-Loss Program on Clinical Control in Obese Adults with Asthma. A Randomized Controlled Trial. Am J Respir Crit Care Med. 2017 Jan;195(1):32-42. 
Miller et al.: Exercise Training in Severe Obesity

23 Jo E, Worts PR, Elam ML, et al. Resistance training during a 12-week protein supplemented VLCD treatment enhances weight-loss outcomes in obese patients. Clin Nutr. 2019 Feb;38(1):372-82.

24 Goodpaster BH, Delany JP, Otto AD, Kuller L, Vockley J, South-Paul JE, et al. Effects of diet and physical activity interventions on weight loss and cardiometabolic risk factors in severely obese adults: a randomized trial. JAMA. 2010 Oct;304(16):1795-802.

25 Wadden TA, Vogt RA, Andersen RE, Bartlett SJ, Foster GD, Kuehnel RH, et al. Exercise in the treatment of obesity: effects of four interventions on body composition, resting energy expenditure, appetite, and mood. J Consult Clin Psychol. 1997 Apr;65(2):269-77.

26 Metcalf B, Rabkin RA, Rabkin JM, Metcalf LJ, Lehman-Becker LB. Weight loss composition: the effects of exercise following obesity surgery as measured by bioelectrical impedance analysis. Obes Surg. 2005 Feb; 15(2):183-6.

27 Coen PM, Tanner CJ, Helbling NL, Dubis GS, Hames KC, Xie H, et al. Clinical trial demonstrates exercise following bariatric surgery improves insulin sensitivity. J Clin Invest. 2015 Jan;125(1):248-57.

28 Hassannejad A, Khalaj A, Mansournia MA, Rajabian Tabesh M, Alizadeh Z. The Effect of Aerobic or AerobicStrength Exercise on Body Composition and Functional Capacity in Patients with BMI $\geq 35$ after Bariatric Surgery: a Randomized Control Trial. Obes Surg. 2017 Nov;27(11):2792-801.

29 Huck CJ. Effects of supervised resistance training on fitness and functional strength in patients succeeding bariatric surgery. J Strength Cond Res. 2015 Mar;29(3):589-95.

30 Daniels P, Burns RD, Brusseau TA, Hall MS, Davidson L, Adams TD, et al. Effect of a randomised 12-week resistance training programme on muscular strength, cross-sectional area and muscle quality in women having undergone Roux-en-Y gastric bypass. J Sports Sci. 2018 Mar;36(5):529-35.

31 Campanha-Versiani L, Pereira DA, Ribeiro-Samora GA, Ramos AV, de Sander Diniz MF, De Marco LA, et al. The Effect of a Muscle Weight-Bearing and Aerobic Exercise Program on the Body Composition, Muscular Strength, Biochemical Markers, and Bone Mass of Obese Patients Who Have Undergone Gastric Bypass Surgery. Obes Surg. 2017 Aug;27(8):2129-37.

32 Dixon JB, Lambert EA, Grima M, Rice T, Lambert GW, Straznicky NE. Fat-free mass loss generated with weight loss in overweight and obese adults: what may we expect? Diabetes Obes Metab. 2015 Jan;17(1):91-3.

33 Donnelly JE, Sharp T, Houmard J, Carlson MG, Hill JO, Whatley JE, et al. Muscle hypertrophy with large-scale weight loss and resistance training. Am J Clin Nutr. 1993 Oct;58(4):561-5.

34 Rantanen T. Muscle strength, disability and mortality. Scand J Med Sci Sports. 2003 Feb;13(1):3-8.

35 Shephard RJ. Maximal oxygen intake and independence in old age. Br J Sports Med. 2009 May;43(5):342-6.

36 Kodama S, Saito K, Tanaka S, Maki M, Yachi Y, Asumi M, et al. Cardiorespiratory fitness as a quantitative predictor of all-cause mortality and cardiovascular events in healthy men and women: a meta-analysis. JAMA. 2009 May;301(19):2024-35.

37 Schwingshackl L, Dias S, Hoffmann G. Impact of long-term lifestyle programmes on weight loss and cardiovascular risk factors in overweight/obese participants: a systematic review and network meta-analysis. Syst Rev. 2014 Oct;3(1):130.

38 Foster-Schubert KE, Alfano CM, Duggan CR, Xiao L, Campbell KL, Kong A, et al. Effect of diet and exercise, alone or combined, on weight and body composition in overweight-to-obese postmenopausal women. Obesity (Silver Spring). 2012 Aug;20(8):1628-38.

39 Lemstra M, Bird Y, Nwankwo C, Rogers M, Moraros J. Weight loss intervention adherence and factors promoting adherence: a meta-analysis. Patient Prefer Adherence. 2016 Aug;10:1547-59.

40 Lee DC, Sui X, Church TS, Lavie CJ, Jackson AS, Blair SN. Changes in fitness and fatness on the development of cardiovascular disease risk factors hypertension, metabolic syndrome, and hypercholesterolemia. J Am Coll Cardiol. 2012 Feb;59(7):665-72.

41 Bellicha A, Ciangura C, Poitou C, Portero P, Oppert JM. Effectiveness of exercise training after bariatric surgerya systematic literature review and meta-analysis. Obes Rev. 2018 Nov;19(11):1544-56.

42 Muschitz C, Kocijan R, Haschka J, Zendeli A, Pirker T, Geiger C, et al. The Impact of Vitamin D, Calcium, Protein Supplementation, and Physical Exercise on Bone Metabolism After Bariatric Surgery: the BABS Study. J Bone Miner Res. 2016 Mar;31(3):672-82.

43 Blumenthal JA, Sherwood A, Gullette EC, Babyak M, Waugh R, Georgiades A, et al. Exercise and weight loss reduce blood pressure in men and women with mild hypertension: effects on cardiovascular, metabolic, and hemodynamic functioning. Arch Intern Med. 2000 Jul;160(13):1947-58.

44 Zhang X, Devlin HM, Smith B, Imperatore G, Thomas W, Lobelo F, et al. Effect of lifestyle interventions on cardiovascular risk factors among adults without impaired glucose tolerance or diabetes: A systematic review and meta-analysis. PLoS One. 2017 May;12(5):e0176436.

45 Donnelly JE, Blair SN, Jakicic JM, Manore MM, Rankin JW, Smith BK; American College of Sports Medicine. American College of Sports Medicine Position Stand. Appropriate physical activity intervention strategies for weight loss and prevention of weight regain for adults. Med Sci Sports Exerc. 2009 Feb;41(2):459-71. 